\title{
Sostenibilidad, justicia ambiental, redes
}

\section{POR SILVIA NONNA $\left(^{*}\right)$}

\author{
Sumario: I. Introducción.- II. Ambiente, justicia, sostenibilidad y de- \\ recho ambiental.- III. Justicia ambiental.- IV. Justicia ambiental y re- \\ des.- V. Reflexiones finales.- VI. Bibliografía.
}

Resumen: el objetivo de este artículo es introducir el tema de la sostenibilidad, su relación con la importancia que representa la regulación ambiental como instrumento para el logro del desarrollo sostenible, presentar la cuestión de la justicia ambiental que propende al trato justo y la participación efectiva de todas las personas hacia el efectivo ejercicio del derecho humano al ambiente sano y la equitativa distribución de los recursos ambientales, destacando el valor de las redes para el reconocimiento del concepto y su desarrollo tanto político como jurídico.

Palabras claves: sostenibilidad - justicia ambiental - redes

\section{Sustainability, environmental justice, networks}

Abstract: the aim of this article is to introduce the concept of sustainability, its relationship with the importance of environmental regulation as an instrument for

(*) Doctora en leyes, Universidad de Buenos Aires. Post Doctora, Facultad de Derecho, Universidad de Buenos Aires. Abogada. Esp. en Recursos Naturales, Facultad de Derecho, Universidad de Buenos Aires. Posgrado en Comercio Internacional de Hidrocarburos, Facultad de Ingeniería, Universidad de Buenos Aires. Posgrado en Derecho Ambiental, Universidad Austral. Environmental Research Fellow, George Washington University, Estados Unidos. Desde 2010 Secretaria Académica, Facultad de Derecho, Universidad de Buenos Aires. Prof. Titular de Derecho de los Recursos Naturales y Protección del Ambiente, Facultad de Derecho, Universidad de Buenos Aires. Prof. Titular de Derecho Ambiental, Universidad Abierta Interamericana. Vicedirectora del Centro de Derecho Ambiental, Facultad de Derecho, Universidad de Buenos Aires. Integrante de la red Just Side. Vicepresidente del Centro de Formación Judicial de la Corte Suprema de la Ciudad de Buenos Aires. Directora de programas y proyectos de investigación y desarrollo. 
achieving sustainable development. To present the issue of environmental justice that tends to fair treatment and the effective participation of all people towards the effective exercise of the human right to a healthy environment and the equitable distribution of environmental resources. Highlighting the value of networks for the recognition of the concept and its political and legal development.

Keywords: sustainability - environmental justice - networks

\section{Introducción}

No cabe duda que el ambiente, en los últimos tiempos, se ha convertido en un tema candente, de actualidad, de debate, de discusión y de preocupación. Es en el último tercio del siglo pasado que la comunidad internacional reacciona ante los cambios evidentes y que se comienza a pensar que es necesario hacer compatibles el crecimiento económico con el correlativo deber de proteger el ambiente.

Surge el concepto del desarrollo sostenible que se va planteando en distintas áreas y necesariamente también se refleja en el derecho.

Han surgido, además, una serie de conceptos, criterios y perspectivas que hay que interpretar de manera armónica y sobre la base que nos brinda un número importante de normas que han consolidado al derecho ambiental como una disciplina en crecimiento, transversal, multi e interdisciplinaria.

Y es el derecho el que nos da un marco para encarar un tema tan vigente como es el de la justicia ambiental, que propende al trato justo y la participación efectiva de todas las personas hacia el efectivo ejercicio del derecho humano al ambiente sano y la equitativa distribución de los recursos ambientales.

Se hace necesario trabajar sobre ese desequilibrio o insostenibilidad, que sabemos afecta a los grupos más vulnerables, que son los que quedan más expuestos a las consecuencias adversas generadas sobre el ambiente, fortaleciendo y haciendo un ejercicio efectivo de los principios de solidaridad y cooperación, pensando en las generaciones futuras.

\section{Ambiente, justicia, sostenibilidad y derecho ambiental}

La sostenibilidad es una construcción a partir de la cual la sociedad, en la última década del siglo $\mathrm{XX}$, ha problematizado condiciones materiales y ha planteado, desarrollado y consolidado ciertos principios éticos y políticos que regulan el acceso a la distribución equitativa de los recursos ambientales.

Esto se ha afianzado a nivel mundial y así es que el derecho ambiental se caracterice por tener una perspectiva global que luego se va consolidando a nivel 
regional, nacional, local. Se trata de un derecho que compartimos, entre todos los pueblos, mujeres y hombres, sin distinción alguna de credos o razas. Porque los problemas ambientales nos atañen a todos y, en mayor o menor medida, antes o más adelante, nos afectan a todos, y, por lo tanto, todos nos tenemos que comprometer a enfrentarlos y fundamental y prioritariamente a evitarlos.

El ordenamiento jurídico fue evolucionando, tanto a nivel internacional como nacional, enfrentando el desafío que significa alcanzar metas imprescindibles. $\mathrm{Y}$ en la búsqueda la "justicia ambiental", el derecho es clave.

También ha evolucionado notablemente la actuación de los operadores jurídicos, cada vez más involucrados en la temática, cada uno desde el rol que le toque asumir ante un conflicto ambiental, o lo que es mejor aún, ante la posibilidad de evitar dicho conflicto.

Se trata de un compromiso que debemos compartir y para el que debemos todos colaborar, con el objetivo de construir una sociedad global viable, que perdure en el tiempo con justicia social.

\section{Justicia ambiental}

Entenderemos la justicia ambiental como un concepto que apunta al trato justo y la participación efectiva de todas las personas, independientemente de su raza, color, origen, bienes o ingresos, en relación con el desarrollo, la implementación y cumplimiento de las normas y las políticas ambientales. Se logrará cuando todos disfrutemos del mismo grado de protección contra los riesgos ambientales y de salud y cuando esté consagrado el equitativo acceso al proceso de toma de decisiones para gozar de un ambiente saludable que nos permita vivir, aprender, trabajar y desarrollarnos.

Considerando al ambiente como un bien común, la justicia ambiental está alcanzando un importante reconocimiento y desarrollo tanto político como jurídico. En este sentido, el derecho ambiental es una sólida base que atraviesa transversalmente instituciones y organizaciones, llegando a todos los ámbitos sociales.

\section{III.1. Antecedentes}

En el siglo pasado, a fines de los setenta, surgieron en los Estados Unidos de Norteamérica grupos ambientalistas que plantearon la prioridad en la consideración de grupos vulnerables de la sociedad y la posibilidad de una calidad de vida aceptable, por sobre la conservación de la vida silvestre (Arnold, 2000). 
Nace, entonces, un movimiento social por la justicia ambiental cuyo principal objetivo es lograr una distribución más equitativa de lo que dan en llamar "cargas y beneficios ambientales". Cargas en cuanto a los costos y los daños que provocan actividades con impacto ambiental, y beneficio ambiental por los servicios que el ecosistema brinda al ambiente.

Esa postura fue extendiéndose, no por una cuestión de imposición o de insistencia por parte de los grupos que la fueran adoptando, sino por la racionalidad que implica considerar lo social, lo ambiental, lo económico, de manera integral y en el marco del desarrollo sostenible, y con justicia.

Un par de décadas después, y habiendo tenido una consideración similar, el tema comenzó a instalarse en Europa. En Reino Unido, las comunidades con menores recursos y posibilidades se ven afectadas por cuestiones como la contaminación del aire, el ruido, el menor acceso al agua, a espacios verdes, con inadecuados servicios sanitarios básicos. La cuestión se va centrando en la situación socioeconómica del grupo afectado.

En América Latina, la consideración de las comunidades originarias se constituye en la base del desarrollo del concepto de justicia ambiental, que luego se extiende a los grupos sociales vulnerables por su situación económica que deriva sin duda en cuestiones que resultan inequitativas.

Y, a nivel global, es muy importante tener en cuenta el principio establecido en la Declaración de Naciones Unidas de Río de Janeiro de 1992(1) de "responsabilidad común pero diferenciada", que es el puntapié para que en todas las negociaciones internacionales se tenga en cuenta, y en la mayoría de las cuales se recoja formalmente en sus textos, como norte para la protección del ambiente como un bien común.

El derecho internacional recoge el concepto de justicia ambiental.

\section{III.2. Aspectos básicos}

Entonces, cuando nos referimos a justicia ambiental, nos referimos a la justicia con sus elementos y aspectos propios, y a lo ambiental también con elementos propios casi exclusivos, que combinamos con el objeto de poder considerar una distribución equitativa de cargas y beneficios ambientales entre todos quienes forman parte de la sociedad, sin hacer distinciones pero, por otro lado y,

(1) Declaración de Río de Janeiro sobre Medio Ambiente y Desarrollo. 1992. Recuperado de https:// www.un.org/spanish/esa/sustdev/agenda21/riodeclaration.htm [Fecha de consulta: 18/03/2020]. 
precisamente, atendiendo a las distintas situaciones y la capacidad de algunos de los integrantes de esa comunidad que resulten ser más vulnerables, y apuntando a la adopción de decisiones adecuadas y "justas".

Y cuando hablamos de justicia ambiental estamos pensando en el acceso a los recursos naturales de manera igualitaria, al aprovechamiento necesario para satisfacer necesidades y obtención de bienes culturales a partir de los naturales. En el uso racional y en la garantía de permitir el mismo uso a las generaciones futuras.

Es muy común encarar el tema de la justica ambiental en relación con casos de contaminación y localización de fuentes que generan riesgo, aunque, en países como el nuestro, también hay que considerar, al momento de hablar de justicia ambiental, los recursos naturales en cuanto a las condiciones de acceso, al uso y al aprovechamiento de ellos, lo que ciertamente pone de manifiesto inequidades dentro de la sociedad al momento de evaluar la distribución de cargas y beneficios.

Resaltando el concepto de desarrollo sostenible como la satisfacción de "las necesidades de la generación presente sin comprometer la capacidad de las generaciones futuras para satisfacer sus propias necesidades" (2), principio rector a nivel mundial. Integra tres variables fundamentales: el desarrollo económico, el desarrollo social y la protección del ambiente.

Por su parte, la participación ciudadana a partir del reconocimiento de las inequidades requiere de un acompañamiento serio y responsable de las autoridades, que a su vez deben constituirse en instituciones sólidas y capacitadas que puedan guiar y garantizar procedimientos que aseguren esa igualdad que se intenta lograr.

Elementos fundamentales de la justicia y, en análisis que se hace en este artículo, de la justicia ambiental, directa y estrechamente relacionado con el derecho ambiental es el derecho a la información y el derecho a la educación ambientales. Principios internacionales recogidos en el derecho internacional y con una

(2) Informe Nuestro futuro común, conocido como Informe Brundland, producido por Comisión mundial creada en 1987 para el desarrollo del medio ambiente, presidida por la exprimera ministra de Noruega Gro Harlem Brundtland. La Comisión Mundial de Medio Ambiente y Desarrollo de Naciones unidas fue creada durante la Asamblea de ONU de 1983. Definición que fue recogida formalmente en el Principio 3 de la Declaración de Río de Janeiro de 1992. Recuperado de https://web. archive.org/web/20111003074433/http://worldinbalance.net/intagreements/1987-brundtland.php [Fecha de consulta: 18/03/2020]. 
consagración constitucional en nuestro país (3), además de regulación general (4) y específica (5) de presupuestos mínimos, que solo necesitaría de una implementación más eficaz.

Si tomamos entonces a la justicia ambiental como un principio, es necesario dotarlo de un marco normativo adecuado, o, en su caso, fortalecer el existente, de modo de asegurar una distribución equitativa de cargas y beneficios entre la sociedad, lo que permitirá garantizar el derecho humano a vivir en un ambiente sano.

\section{III.3. Sociología ambiental}

Ya he comentado que la evidencia de problemas ambientales que alteran la vida en el planeta y, en consecuencia, las relaciones humanas, se presenta como un panorama complejo e integral que requiere de la participación de distintas ciencias, entre ellas la sociología en el estudio del sistema de relaciones entre la sociedad y el ambiente, fundamentalmente de la interrelación del ser humano con su entorno y de cómo el ambiente influye en el comportamiento humano (Nonna, 2019).

Dice el sociólogo Gómez Tagle López(6) que "La sociología ambiental tiene entonces como uno de sus propósitos facilitar la reapropiación social de la naturaleza, no en términos de la explotación de la que puede ser objeto, sino de la valoración de su potencial ecológico productivo (...)".

Sobre el final de los años 70 los sociólogos norteamericanos Riley Dunlap y William Catton formalizaron unas primeras reflexiones sobre la "sociología ambiental" y propusieron algunas pautas para relacionar la sociedad y el ambiente,

(3) Artículo 41 de la Constitución Nacional: “Todos los habitantes gozan del derecho a un ambiente sano, equilibrado, apto para el desarrollo humano y para que las actividades productivas satisfagan las necesidades presentes sin comprometer las de las generaciones futuras; $y$ tienen el deber de preservarlo. El daño ambiental generará prioritariamente la obligación de recomponer, según lo establezca la ley. Las autoridades proveerán a la protección de este derecho, a la utilización racional de los recursos naturales, a la preservación del patrimonio natural y cultural y de la diversidad biológica, y a la información y educación ambientales. Corresponde a la Nación dictar las normas que contengan los presupuestos mínimos de protección, y a las provincias, las necesarias para complementarlas, sin que aquéllas alteren las jurisdicciones locales. Se prohíbe el ingreso al territorio nacional de residuos actual o potencialmente peligrosos, y de los radiactivos".

(4) Ley 25.675 de Presupuestos Mínimos para la Política Ambiental. Sancionada el 06/11/2002. Promulgada parcialmente, Decreto 2413/02, 27/11/2002. Publicada en el Boletín Oficial el 28/11/2002.

(5) Ley 25.831 de Información Pública Ambiental. Sancionada el 26 de noviembre 2003. Promulgada el 6 de enero de 2004. Publicada el 7 de enero de 2004.

(6) https://www.insumisos.com/lecturasinsumisas/Sociologia\%20ambiental.pdf [Fecha de consulta: $29 / 08 / 2020]$. 
que luego se considerarán como el Nuevo Paradigma Ecológico. Plantearon el Paradigma de la Excepcionalidad Humana - $\mathrm{PEH}-$, que se basa en una visión antropocéntrica de la relación existente entre la sociedad y la naturaleza.

Al parecer estaríamos ante una visión moderna y que ahora suena muy razonable y en general es más que aceptable. Sin embargo, el tema ya había sido planteado y podemos buscar orígenes de la disciplina remontándonos al siglo $\mathrm{V}$ antes de Cristo.

En efecto, Hipócrates, quien iniciara la escuela del determinismo ambiental, sostuvo:

Cuando una raza habita en un áspero país montañoso, a una altitud considerable con unas lluvias cuantiosas y con marcadas diferencias entre estaciones, entonces sus gentes serán de gran talla, bien acostumbrados a la audacia y la valentía y con no poca ferocidad y brutalidad en su carácter. Por otra parte, en tierras bajas, sofocantes, con prados, son más flemáticos que coléricos. La valentía y la audacia no son parte de su carácter, aunque se pueden adquirir con la adecuada formación (Lemkow y Spluga, 2017, p. 67).

Varios siglos después, en el XIII, Santo Tomás de Aquino, refiriéndose a la fuerza necesaria para la guerra, decía que: "las gentes que viven cerca del sol y están resecados por el calor excesivo tienen un intelecto más agudo, y es cierto, pero tienen menos sangre y por consiguiente no tienen constancia en cuanto a la confianza en ellos mismos. Por otro lado, las tribus nórdicas, lejos de los abrasadores rayos del sol, son ciertamente más estúpidos pero siempre están a punto para la guerra" (Lemkow y Spluga, 2017, p. 22).

Ambos autores están refiriéndose a las relaciones entre el ambiente y el hombre, las ventajas y desventajas de acuerdo con la actividad o el por qué estamos considerando la capacidad de la gente.

Montesquieu, en el siglo XVII/XVIII, resulta ser el exponente más conocido del determinismo ambiental. Sostuvo que "el clima, y en menor medida el tipo de suelo configuran el carácter o personalidad de un pueblo o nación dados. Las características de esa personalidad determinan, a su vez, la estructura social y finalmente es la estructura social la que determina el tipo de leyes y la legislación del país. Los climas distintos que han dado lugar a los distintos modos de vida han formado los diversos tipos de leyes" (Lemkow y Spluga, 2017, p. 26).

La escuela de determinismo ambiental fundada por Hipócrates pasó luego un período de oscuridad y ausencia. Y surgen detractores de esa posición. 
En los 50 del siglo pasado, el sociólogo Otis Dudley Duncan estudió la población negra de Chicago y se dedicó a demostrar que la ecología humana es una extensión de la disciplina de su especialidad. Desarrolló un modelo sobre la base de lo que identificó como funciones generales del entorno en el que nos desarrollamos: espacio vital, un lugar para desperdiciar desechos y un depósito de recursos naturales, lo que lo lleva a la conclusión de que todo lo que nos rodea proviene del medio. Funciones que en general separamos, ya que no queremos vivir cerca de donde dejamos nuestros desechos ni donde estamos realizando actividades mineras o cortando árboles. Afirmó también que las cosas que usamos de alguna manera provienen de la naturaleza, que transformamos a través de la tecnología que se fue desarrollando.

Algunos otros autores profundizaron el tema, como José María Aranda Sánchez, que identifica distintas escuelas o tendencias. Para Aranda Sánchez (2013) existen varias perspectivas: 1) materialista marxista, tendencia con historia y consolidación representada por la contraposición entre capital y ecología; 2) ecología profunda, que elimina el antropocentrismo y se asienta en la idea de interdependencia sociedad naturaleza; 3 ) ecofeminismo, posición radical con perspectiva de género que ha llegado a representar una crítica de fondo al capitalismo y al sistema patriarcal, al impulsar una corriente ideológica que cuestiona la posibilidad de la sustentabilidad mientras los hombres tengan el poder; 4) enfoque de la ecología humana crítica acompañado de la teoría de la acción comunicativa de Habermas, que aporta un paradigma diferente al plantear las cuestiones ambientales para la sociedad en términos de dilemas; 5) orientación constructivista, con su análisis de la realidad social como una construcción que implica una parte objetiva, las estructuras sociales, y una subjetiva, que es la visión particular de los sujetos, en una amalgama que permite superar las visiones "realista" e "idealista".

Desde que Hipócrates fundara la escuela del determinismo ambiental, a la fecha, mucho ha pasado y mucho ha cambiado la sociedad en su visión y reconocimiento de la relevancia del ambiente como bien común, como así también en la necesidad de protegerlo. Grandes esfuerzos se han hecho y muchos más son necesarios para alcanzar el desarrollo sostenible, y la necesidad de garantizar el equilibrio y la justicia socio ambiental que considere el crecimiento económico, la preservación del ambiente y el bienestar social con Justicia Ambiental.

\section{Justicia ambiental y redes}

\section{IV.1. Importancia de las redes}

Si tomamos en cuenta que los movimientos surgidos a partir de los años setenta del siglo pasado son los que fueron instalando y consolidando el tema de la 
justicia social, extendiéndose a nivel global, cuánto más rápido y eficaz hubiera resultado dicho impulso con la facilidad que ofrecen las redes tan desarrolladas en la actualidad.

Desde hace un tiempo las redes vienen cumpliendo funciones que, en cierta manera, corresponderían a los gobiernos, y lo hacen sin la participación de ellos, en algunos casos basándose en que hay una razonable transferencia de responsabilidad de lo público a la sociedad civil, y en otros casos tomando una posición intransigente de extremo que puede interpretarse como de avasallamiento.

Podemos ver en las redes reclamos reales y justos, que convocan a la participación y a la suma de esfuerzos hacia el bien común, propuestas concretas de criterios acertados, que convocan a afectados y actores involucrados, impulso de políticas públicas, de normativa adecuada, que requieren de la intervención de los tribunales, y en definitiva apuntan a la justicia ambiental.

Las redes son importantes, porque crean conciencia, porque informan, porque convocan, porque entusiasman, porque muestran realidades, porque acercan a los involucrados y a quienes se comprometen, aunque no sean parte de ese grupo afectado, porque buscan la cooperación, porque crean comités y grupos de alerta, porque impulsan foros de intercambio, porque comparte experiencias, porque visibilizan problemas y tratan de proponer soluciones, en definitiva, porque nos mantienen al tanto.

Podría citar varios ejemplos, pero no es mi intención hacerlo en el contexto de lo que quiero presentar, hay ejemplos para destacar y otros que no han logrado cumplir su objetivo, hay posiciones extremas, hay impulsores y detractores. Valga solo esta introducción para introducir el tema y para comentar los objetivos y las tareas que viene desarrollando una red iberoamericana que ha planteado el tema de la justicia socio ambiental y la necesidad de crear una herramienta que ayude a la toma de decisiones.

\section{IV.2. Just Side - Una red iberoamericana por la justicia ambiental}

Políticas ambientales que se planeen con objetivos justos requerirán de la identificación y el mapeo de las injusticias sobre las que se intente trabajar con el objetivo de resolverlas, como así también la participación de quienes forman parte del grupo involucrado, y decisiones consensuadas y planificadas.

En esa línea de ideas, la red Just Side se ha conformado con el objeto de formular propuestas a partir de la identificación de algunas injusticas sociales en Iberoamérica y aportar una herramienta útil para la planificación de políticas públicas, a través de una experiencia que será acotada por el alcance de la investigación, pero, 
a la vez, repetible a futuros casos. Para ello, un grupo de expertos de varias universidades iberoamericanas, del que participa Argentina (7), liderados por la portuguesa Universidad de Coimbra, ha encarado la tarea de desarrollar un programa de 4 años en el marco del Programa Iberoamericano de Ciencia y Tecnología para el Desarrollo-CYTED (8).

El CYTED es un programa internacional de cooperación científica y tecnológica, multilateral, de ámbito iberoamericano con carácter horizontal, orientado al desarrollo y que cubre todas las fases de la I+D+I(9) bajo una misma coordinación, desde la cooperación en investigación básica y aplicada hasta la cooperación en desarrollo e innovación. Su objetivo principal es contribuir al desarrollo armónico y sostenible de la región Iberoamericana mediante el establecimiento de mecanismos de cooperación entre grupos de investigación de las universidades, centros de I+D y empresas innovadoras de los países iberoamericanos, que pretenden la consecución de resultados científicos y tecnológicos transferibles a los sistemas productivos y a las políticas sociales.

En línea con sus objetivos, y a partir de una propuesta de la Universidad de Coimbra (10), CYTED aprobó el proyecto de red Just Side(11) del que forman parte Argentina, Brasil, Chile, Costa Rica, España, México, Portugal, Uruguay en una primera conformación a la que se ha sumado recientemente Cuba.

La red Just Side, que comenzó a funcionar en 2017, propone realizar un análisis jurídico de los datos territoriales, ambientales y sociales a partir de una visualización geoespacializada, con la finalidad de obtener información sobre injusticias socioambientales que pueda servir como base científica para la adopción o corrección de políticas públicas.

(7) Las profesoras doctoras Silvia Nonna y Leila Devia, titulares de Derecho de los Recursos Naturales y Protección del Ambiente de la Facultad de Derecho de la Universidad de Buenos Aires, investigadoras del Instituto Ambrosio Gioja de esa facultad, integran la red como investigadoras responsable y asociada respectivamente.

(8) CYTED es un Programa Iberoamericano de Ciencia y Tecnología para el Desarrollo (CYTED), creado en 1984 mediante un Acuerdo Marco interinstitucional suscrito por 19 países de América Latina, España y Portugal. Desde 1995, el Programa CYTED se encuentra formalmente incluido entre los programas de cooperación dependientes de las Cumbres Iberoamericanas de Jefes de Estado de la Comunidad Iberoamericana de Naciones.

(9) I+D+I. Investigación, desarrollo e innovación, concepto que surge en el contexto del estudio complementario y relacionada de ciencia, tecnología y sociedad. Que supera el concepto de investigación y desarrollo I+D.

(10) Alexandra Aragon. Recuperado de https://www.youtube.com/watch?v=4gcIdN3Cduk\&feat ure=youtu.be

(11) Recuperado de http://www.cyted.org/es/just-side 
El desafío de la red, además, es el de elaborar/formular una metodología de desarrollo y utilización de una herramienta basada en una infraestructura de datos en soporte digital que ayude a apoyar las decisiones, para promover y fortalecer las políticas públicas, para hacer frente a los desafíos sociales, ambientales, económicos, jurídicos y democráticos.

Los resultados de la investigación, como también la herramienta a utilizar para realizarla, estarán luego disponibles para los gobiernos de los países iberoamericanos, con la intención de contribuir, en el plan académico, con la consolidación del área específica del "geo derecho". Y al mismo tiempo, por otro lado, en el plano político y social para la mejoría de la eficacia y justicia de las políticas públicas con incidencia territorial (12).

Se estudiarán 16 casos específicos(13) en diferentes territorios en los que sea por la propia idiosincrasia social o por resultados de políticas públicas incorrectas o demoradas, se identifican situaciones disvaliosas o dañosas, tanto desde el punto de vista económico como ambiental, para la población involucrada (14).

\section{IV.2.1. Objetivos de la red Just Side}

La red Just Side plantea como objetivo general el de incentivar el intercambio de conocimientos y la cooperación entre diferentes grupos de I+D (investigación y desarrollo) para el desarrollo abierto local de infraestructuras de software para promover la justicia en el territorio y la sostenibilidad de las políticas públicas para que las regiones y los Estados puedan cumplir con los objetivos de desarrollo sostenible asumidos en la Agenda 2030 de la ONU(15).

Se pretende colaborar con la planificación de algunas de las acciones incluidas como directrices de la Agenda 2030 difundiendo el papel que pueden representar las infraestructuras de datos digitales, a la vez que se reconozca la necesidad de crear la capacidad institucional para la construcción de bases de datos y

(12) El geoportal Just Side soportará la infraestructura abierta y regional de datos espaciales apta para una aplicación de ciencia abierta en la nube. La cartografía avanzada que resulta de la superposición de múltiples capas "layers" de información geográfica, ambiental y social, así como los principios, recomendaciones y buenas prácticas de integración de la justicia socio-ambiental en las políticas públicas de los países integrantes de la Red quedarán disponibles en el sitio Just Side.

(13) 16 era el número en origen del proyecto, que se ha incrementado en 2 más con la participación de Cuba.

(14) Ver punto VI, los casos argentinos.

(15) Recuperado de http://www.ar.undp.org/content/argentina/es/home/post-2015/sdg-overview.html 
el suministro de información de calidad, accesible, oportuna, fiable, detallada y georreferenciada(16).

Uno de los objetivos específicos es el de potenciar sinergias y la coordinación de las líneas de I + D de los miembros originales de la red, extendiéndolo a quienes luego vayan sumándose para ampliarla. El desafío es desarrollar una infraestructura abierta y regional de datos espaciales apta para una aplicación de ciencia abierta en la nube.

La Ciencia que será desarrollada es el "geo derecho", entendiéndolo como un análisis jurídico de los datos territoriales, ambientales y sociales en forma de visualización y presentación geoespacializada a fin de obtener información jurídicamente relevante que pueda servir como base científica para la adopción de políticas públicas.

La red tiene como misión la interconexión entre las nuevas tecnologías para crear infraestructuras de datos digitales y el derecho, para crear herramientas de apoyo a la decisión y participación. Un objetivo fundamental de la red es el de crear el potencial para el uso de los sistemas de datos para la promoción de la justicia territorial, la protección del ambiente, el uso eficiente de los recursos naturales, el uso sostenible del suelo, la redistribución equitativa, entre otros.

El objetivo que mayores desafíos propone la red es la colaboración en el refuerzo de la base científica de las decisiones que puedan aportar a la planificación de políticas públicas sólidas cuyos resultados se cristalicen en el fortalecimiento de la ciudadanía y de la consciencia ambiental. Con el soporte de datos científicos puede garantizarse una mayor legitimidad a las políticas, mejorando la aceptabilidad y legitimación de las decisiones, y pueden prevenirse conflictos y fortalecer la sostenibilidad.

\section{IV.2.2. Socios de la red}

Algo que corresponde destacar y que le da una especial importancia a la red es la participación de socios en diferentes áreas científicas, como el derecho, la geografía, la geología, las tecnologías de información, las políticas públicas, la ciencia política, la administración pública, y en diferentes contextos geopolíticos.

Distintas especialidades que requieren de socios especialistas, tanto del sector académico como incluso del empresarial.

(16) Como surge de los párrafos 48 y 57 de la Agenda. 
Son socios del área académico/científica, en distintas especialidades:

Derecho: Facultad de Derecho de la Universidad de Buenos Aires (Argentina), Universidad de La Rioja (España), Universidad de Santa Catarina (Brasil), Universidad de Costa Rica (Costa Rica), Instituto Jurídico de la Facultad de Derecho de la Universidad de Coimbra (Portugal).

Información y georreferenciación: Universidad Nacional Autónoma de México (México), Departamento de Ingeniería Geográfica de la Facultad de Ingeniería de la Universidad de Santiago de Chile (Chile), Facultad de Ciencias UDELAR (Uruguay), Centro de Estudios de Geografía y Ordenamiento del Territorio de la Universidad de Coimbra (Portugal).

Social: Unidad de Investigación en Gobernanza, Competitividad y Políticas Públicas de la Universidad de Aveiro (Portugal).

Por su parte, los socios empresariales Geodireito (17) y Asociación GV SIG(18) se constituyen en apoyo fundamental por la especificidad del proyecto en cuanto a la organización de la red, la visualización del proyecto, a la utilización del mapeo y la representación cartográfica y la geo referenciación de datos.

Téngase en cuenta que se utilizará la producción de mapas con múltiples capas como forma de visualización avanzada, lo que contribuirá a la comprensión de fenómenos sociales complejos, identificando condiciones de vivienda, acceso a agua potable, condiciones básicas de salubridad y condiciones sociales en general como acceso a medios de transporte, a hospitales, a educación, a trabajo, entre otras. Fenómenos relacionados entre sí que es importante poder visualizarlos en conjunto para contribuir a la identificación de causas y efectos como así también de factores de peligrosidad y riesgo (19).

\section{IV.2.3. Resultados esperados}

La red aspira a aportar conocimientos en georreferenciación a partir de datos obtenidos organizados transversalmente y de manera integrada sumando disciplinas como derecho, economía, geografía y geología, sociología, gobernanza y políticas públicas.

(17) Recuperado de http://geodireito.com.br/

(18) Recuperado de www.gvsig.com/es

(19) Recuperado de https://ejscreen.epa.gov/mapper 
Se propone investigar y recopilar datos, poniéndolos a disposición a través de técnicas de interconexión, organizando la información y complementándola con un actualizado análisis jurídico/normativo.

La red Just Side pretende crear una metodología de desarrollo y utilización de una herramienta basada en una infraestructura de datos en soporte digital para apoyar las decisiones, para promover y fortalecer las políticas públicas, para hacer frente a los desafíos sociales, ambientales, económicos, jurídicos y democráticos.

Los estudios de caso permitirán probar la precisión, alcance, eficiencia y eficacia de las técnicas de "geo derecho", que permitan esbozar patrones geoespaciales para identificar injusticias ambientales y exclusión territorial, interpretándolas a la luz de políticas públicas para generar orientaciones generales compartibles y repetibles.

Se formulará un conjunto de "principios de integración de técnicas de geo derecho en las políticas de ordenamiento territorial y de ambiente y demás políticas sectoriales", una guía metodológica de condiciones de utilización, áreas potenciales de aplicación, etapas principales, métodos, procedimientos y procedimientos principios de espacialización de datos y comunicación de información.

A su vez, se prevén beneficios con la implementación de la propuesta en la región iberoamericana a través del desarrollo de nuevas herramientas para el monitoreo, la prospectiva y la construcción de escenarios de la justicia tanto presente como futura y a nivel territorial, social y ambiental.

El desafío de la red es el de crear el potencial para el uso de los sistemas de datos para la promoción de la justicia territorial, el uso eficiente y racional de los recursos naturales, la protección del ambiente, el uso sostenible del suelo y la redistribución equitativa de ingresos. Se apunta a promover la justicia territorial y la sostenibilidad de las políticas públicas, cumpliendo los objetivos de desarrollo sostenible de la ONU.

\section{IV.2.4. Casos que Argentina estudia en el marco de la red}

Los nueve países participantes (20) aportarán datos sobre casos que fueron consensuados entre todos los participantes. Se trata de dos casos por país, que se estudiarán de manera multi e interdisciplinaria, con utilización de datos existentes

(20) Ocho originales que, con la incorporación de Cuba a partir del segundo año del proyecto, son nueve. 
y previendo la producción de nuevos datos georreferenciados y analizados desde la óptica del geo derecho.

Para Argentina se seleccionaron dos casos, el de la cuenca Matanza Riachuelo, y el de los bosques nativos en comunidades originarias.

\section{1) El caso de la Cuenca Matanza Riachuelo}

Valga recordar que la Cuenca Matanza Riachuelo (CMR) es una de las cuencas más contaminadas del mundo, alcanza niveles críticos en los cursos medio y bajo del río y tiene consecuencias directas sobre la salud y calidad de vida de quienes habitan la zona. Podemos considerarlo como un caso representativo de contaminación y consecuentemente de injusticia ambiental.

Se han elaborado planes. Se han solicitado fondos internacionales. Se han creado organismos. Incluso la Suprema Corte de Justicia de la Nación ha tomado activa participación/intervención en el tema. Se está trabajando, aunque el problema persiste. La cuenca pone de manifiesto una creciente degradación ambiental y una histórica falta de abordaje integral.

En la cuenca coexisten distintos niveles de densidad poblacional, actividades productivas y ecosistemas, lo que se traduce en diferentes situaciones ambientales de las que dan cuenta los relevamientos del ordenamiento territorial y los monitoreos de la calidad de sus aguas.

La CMR abarca una superficie de aproximadamente $2.300 \mathrm{~km} 2$ y tiene una población de aproximadamente 6 millones de personas. La extensión del sistema hídrico integrado por los ríos Matanza y Riachuelo es de $64 \mathrm{~km}$. Resulta necesario implementar mecanismos que permitan ajustar la política y la gestión ambiental de este vasto territorio a las diversidades propias de cada área.

En este orden de ideas, contar con datos es un primer paso, organizarlos y sistematizarlos un siguiente paso, volcar los datos en mapas identificados por ítems o temas y, finalmente, superponer esos mapas que reflejen los datos, permitirá una visión integral y comparable de la información.

La ACUMAR, a través del PISA 2010 actualizado en 2016 ha recuperado documentación existente, ha ido incorporando nuevos y actuales datos y hoy se cuenta con información mapeable sobre la que se está trabajando(21).

(21) Recuperado de www.acumar.gob.ar 


\section{2) Bosques y comunidades originarias}

También se planea estudiar la situación de las comunidades originarias en su relación con los bosques nativos que habitan y la efectividad de la Ley de Presupuestos Mínimos 26.331 (22) de protección de bosques nativos.

Téngase presente que tanto la Constitución Nacional como la Ley de bosques nativos señalan la necesidad de respetar la cultura ancestral de las comunidades originarias, y en lo que se analizará, fundamentalmente a través de la promoción de la conservación, restauración y uso responsable de los servicios y productos de los bosques nativos, apuntando a los servicios ambientales que brindan, teniendo como premisa que la mejor manera de cuidar los bosques es fortaleciendo a las comunidades que los habitan.

La autoridad ambiental nacional está implementando planes integrales comunitarios (PIC) con el objetivo de apoyar y complementar la implementación de las políticas nacionales de manejo sostenible de los bosques nativos en el marco la Ley de bosques, en especial respecto de las pequeñas comunidades.

A través de los PIC se planifican y desarrollan, en conjunto con los beneficiarios, actividades con especial énfasis en servicios, prácticas sostenibles y herramientas específicas que mejoren la calidad de vida de las comunidades directamente relacionadas al bosque nativo. La planificación apunta, estratégicamente, a la producción sustentable y la comercialización de bienes y servicios provenientes de los bosques nativos (23).

Para ello, el proyecto Bosques Nativos y Comunidad ha realizado una evaluación social y ambiental de la región chaqueña, datos con los que se trabajará para mapear distintos indicadores y criterios para luego preparar la comparación de capas.

\section{Reflexiones finales}

El derecho humano a vivir en un ambiente sano, equilibrado y que permita el desarrollo de las actividades presentes sin comprometer la satisfacción de las necesidades de las generaciones futuras es un tema muy presente hoy en la sociedad.

(22) Ley 26.331. B.O. 26/12/2007. Presupuestos Mínimos para la Protección Ambiental de los Bosques Nativos. Recuperado de http://servicios.infoleg.gob.ar/infolegInternet/anexos/135000-139999/136125/ norma.htm

(23) Las acciones se focalizan inicialmente en las regiones del parque chaqueño en las provincias de Salta, Santiago del Estero y Chaco. 
Es un tema candente, de actualidad, de debate, de búsqueda de consensos y de preocupación.

La sostenibilidad es una construcción a partir de la cual la sociedad ha problematizado condiciones materiales y ha planteado, desarrollado y consolidado ciertos principios éticos y políticos que regulan el acceso a la distribución equitativa de los recursos ambientales, basada en principios de solidaridad y cooperación, buscando la justicia social.

El crecimiento de la población, la concentración en áreas determinadas, las diferentes necesidades de consumo, la accesibilidad a bienes básicos, producen alteraciones en el ambiente y se generan inequidades o injusticias sociales. Son los sectores sociales vulnerables los más afectados y al mismo tiempo son los sectores que no cuentan ni pueden acceder a las herramientas necesarias para morigerar las consecuencias ambientales adversas.

La justicia ambiental apunta al trato justo y la participación efectiva de todas las personas, independientemente de su raza, color, origen, bienes o ingresos, en relación con el desarrollo, la implementación y cumplimiento de las normas y las políticas ambientales, y se logrará cuando todos disfrutemos del mismo grado de protección contra los riesgos ambientales y de salud y cuando esté consagrado el equitativo acceso al proceso de toma de decisiones para gozar de un ambiente saludable que nos permita vivir, aprender, trabajar y desarrollarnos.

Destaco la importancia de las redes, que crean conciencia, que informan, que convocan, que acercan a los involucrados, que crean comités y grupos de alerta, que comparten experiencias, que muestran realidades y visibilizan problemas y al mismo tiempo proponen soluciones, que ayudan a tomar decisiones y a planificar.

Como aporte, la red Just Side, de la que participa Argentina, se constituye en una herramienta para identificar injusticas sociales en Iberoamérica. El objetivo de la propuesta es realizar un análisis jurídico de los datos territoriales, ambientales y sociales a partir de una visualización geoespacializada a fin de obtener información sobre injusticias socioambientales que pueda servir como base científica para la adopción o corrección de políticas públicas.

Se espera contribuir en el desarrollo y la consolidación del área específica del "geo derecho", formulando y testeando una metodología de desarrollo y utilización de una herramienta basada en una infraestructura de datos en soporte digital que ayude a apoyar las decisiones, para promover y fortalecer las políticas públicas, para hacer frente a los desafíos sociales, ambientales, económicos, jurídicos y democráticos. 
El desafío de la red es el de crear el potencial para el uso de los sistemas de datos para la promoción de la justicia territorial, el uso eficiente y racional de los recursos naturales, la protección del ambiente, el uso sostenible del suelo, la redistribución equitativa de ingresos.

Se apunta a promover la justicia territorial y la sostenibilidad de las políticas públicas, cumpliendo los Objetivos de Desarrollo Sostenible de la Organización de las Naciones Unidas.

\section{Bibliografía}

Aranda Sánchez, J. (2013). Principales desarrollos de la sociología ambiental. México: Centro de Investigación en Ciencias Sociales y Humanidades, Universidad Autónoma del Estado de México.

Arnold, C. (2000). Land Use Regulation and Environmental Justice. Environmental Law Reporter.

Nonna, S. (2019). Desarrollo Sostenible. Sociología ambiental y justicia socio ambiental. Revista Temas de Derecho de Civil, Persona y Patrimonio. Buenos Aires: ERREPAR.

Lemkow, L. y Espluga, J. (2017). Sociología Ambiental. Barcelona: Icaria-Antrazyt Editora.

Fecha de recepción: 31-03-2020

Fecha de aceptación: 18-06-2020 